\title{
Type 2 Diabetes Mellitus, Metabolic Syndrome, Lipids
}

\author{
Orit Pinhas-Hamiel \\ Pediatric Endocrinology and Diabetes Unit, Edmond and Lily Safra Children's Hospital, Sheba Medical Center, \\ Ramat-Gan and Maccabi Juvenile Diabetes Center, Raanana, Sackler School of Medicine, Tel-Aviv University, \\ Israel
}

Identifying the genetic variants that increase the risk of type 2 diabetes mellitus (T2DM) in humans continues to be a challenge. In the search to identify diabetes susceptibility genes, genome-wide association (GWA) studies have revealed not only new loci but also new possible underlying mechanisms and hypotheses of T2DM pathogenesis. The impact of T2DM during childhood on the early development of diabetes complications, including abnormalities in carotid structure and function, as well as the development and progression of microalbuminuria in children with T2DM, has been the subject of a number of recent studies. Research on metformin treatment as protection against cancer incidence and mortality in patients with T2DM is presented.

Longitudinal follow-up has demonstrated a high risk of premature mortality among subjects who had features of the metabolic syndrome (MetS) during their childhood. A number of articles published this year have highlighted dilemmas in definitions of the MetS, as well as revelations and challenges [1, 2]. Rapid weight gain in newborns with intrauterine growth retardation and precocious puberty has both been found to be associated with the development of the Mets.

Hyperlipidemia among children and adolescents continues to be on the front line, as expressed in an editorial entitled 'a decade of progress' [3] and as manifested by the emergence of new drugs for children with familial hyperlipidemia. Hyperlipidemia has been associated to another 'hot topic', vitamin D. Finally, we discuss the impact of the contraception pill, now celebrating its 50th anniversary on lipids.

\section{New paradigms}

\section{Type 2 diabetes risk alleles are associated with reduced size at birth}

Freathy RM, Bennett AJ, Ring SM, Shields B, Groves CJ, Timpson NJ, Weedon MN, Zeggini E, Lindgren CM, Lango H, Perry JR, Pouta A, Ruokonen A, Hypponen E, Power C, Elliott P, Strachan DP, Jarvelin MR, Smith GD, McCarthy MI, Frayling TM, Hattersley AT

Genetics of Complex Traits, Institute of Biomedical and Clinical Science, Peninsula Medical School, Exeter, UK Diabetes 2009;58:1428-1433

Background: Low birth weight is associated with an increased risk of T2DM. The mechanisms underlying this association are unknown and may represent intrauterine programming or two phenotypes of one genotype. The fetal insulin hypothesis proposes that common genetic variants that reduce insulin secretion or action may predispose to both T2DM and also reduce birth weight, since insulin is a key fetal growth factor.

Methods: Single-nucleotide polymorphisms (SNPs) at five recently identified T2DM loci (CDKAL1, CDKN2A/B, HHEX-IDE, IGF2BP2, and SLC30A8) were genotyped in 7,986 mothers and 19,200 offspring white Europeans. The association between maternal or fetal genotype at each locus and birth weight of the offspring was tested.

Results: T2M risk alleles at the CDKAL1 and HHEX-IDE loci were associated with reduced birth weight when inherited by the fetus (21 g [95\% CI 11-31], $\mathrm{p}=2 \times 10^{-5}$, and $14 \mathrm{~g}$ [4-23], $\mathrm{p}=0.004$, lower birth weight per risk allele, respectively). The $4 \%$ of offspring carrying four risk alleles at these two loci were 80 g (95\% CI 39-120) lighter at birth than the $8 \%$ carrying none $\left(\mathrm{p}_{\text {trend }}=5 \times 10^{-7}\right)$. There were no associations between birth weight and fetal genotypes at the three other loci or maternal genotypes at any locus. 
Conclusions: These findings provide robust evidence that common disease-associated variants can alter size at birth directly through the fetal genotype.

Low birth weight is an established risk factor for the development of T2DM. An explanation based on the insulin resistance model suggests that metabolic adaptation to fetal undernutrition persists into adult life. According to this theory, fetal programming, which basically expresses plasticity for shortterm survival, results later in adult life in a range of metabolic abnormalities. Alternatively, according to the fetal insulin hypothesis, the same genetic variants that reduce insulin secretion or insulin sensitivity also reduce birth weight. Two observations support this hypothesis. First, patients with monogenic neonatal diabetes have reduced birth weights. Second, offspring of fathers who develop diabetes later in life have, on average, lower birth weights than those born to fathers who do not develop diabetes. This is consistent with the fact that the fetus inherits about $50 \%$ of the father's genetic predisposition to diabetes, as well as the genetic predisposition to reduced fetal growth. Interestingly, the authors discuss that maternal genotypes cannot help us understand such inheritance, since the effect of maternal genes is confounded by that of maternal hyperglycemia on birth weight.

To test the fetal insulin hypothesis, the authors investigated the relationship between the size of the fetus at birth and five known T2DM variants identified through genomic-wide association. Two risk alleles that have been associated with reduced $\beta$-cell function, CDKAL1 and HHEX-IDE, were associated with reduced birth weight. The authors hypothesized that reduced fetal insulin secretion in utero results in reduced fetal size at birth, and subsequently leads to T2DM. The example presented of two phenotypes for a common genotype is compelling. Nevertheless, in the editorial accompanying this article [4], Meier discusses the idea that the pathogenesis of T2DM likely involves a combination of several mechanisms with varying contributions in different people.

\section{Genetic variation in GIPR influences the glucose and insulin responses to an oral glucose challenge}

Saxena R, Hivert MF, Langenberg C, Tanaka T, Pankow JS, Vollenweider P, Lyssenko V, Bouatia-Naji N, Dupuis J, Jackson AU, Kao WH, Li M, Glazer NL, Manning AK, Luan J, Stringham HM, Prokopenko I, Johnson T, Grarup N, Boesgaard TW, Lecoeur C, Shrader P, O’Connell J, Ingelsson E, Couper DJ, Rice K, Song K, Andreasen CH, Dina C, Kottgen A, Le Bacquer O, Pattou F, Taneera J, Steinthorsdottir V, Rybin D, Ardlie K, Sampson M, Qi L, van Hoek M, Weedon MN, Aulchenko YS, Voight BF, Grallert H, Balkau B, Bergman RN, Bielinski SJ, Bonnefond A, Bonnycastle LL, Borch-Johnsen K, Bottcher Y, Brunner E, Buchanan TA, Bumpstead SJ, Cavalcanti-Proenca C, Charpentier G, Chen YD, Chines PS, Collins FS, Cornelis M, Crawford GJ, Delplanque J, Doney A, Egan JM, Erdos MR, Firmann M, Forouhi NG, Fox CS, Goodarzi MO, Graessler J, Hingorani A, Isomaa B, Jorgensen T, Kivimaki M, Kovacs P, Krohn K, Kumari M, Lauritzen T, Levy-Marchal C, Mayor V, McAteer JB, Meyre D, Mitchell BD, Mohlke KL, Morken MA, Narisu N, Palmer CN, Pakyz R, Pascoe L, Payne F, Pearson D, Rathmann W, Sandbaek A, Sayer AA, Scott LJ, Sharp SJ, Sijbrands E, Singleton A, Siscovick DS, Smith NL, Sparso T, Swift AJ, Syddall H, Thorleifsson G, Tonjes A, Tuomi T, Tuomilehto J, Valle TT, Waeber G, Walley A, Waterworth DM, Zeggini E, Zhao JH, Illig T, Wichmann HE, Wilson JF, van Duijn C, Hu FB, Morris AD, Frayling TM, Hattersley AT, Thorsteinsdottir U, Stefansson K, Nilsson P, Syvanen AC, Shuldiner AR, Walker M, Bornstein SR, Schwarz P, Williams GH, Nathan DM, Kuusisto J, Laakso M, Cooper C, Marmot M, Ferrucci L, Mooser V, Stumvoll M, Loos RJ, Altshuler D, Psaty BM, Rotter Jl, Boerwinkle E, Hansen T, Pedersen O, Florez JC, McCarthy MI, Boehnke M, Barroso I, Sladek R, Froguel P, Meigs JB, Groop L, Wareham NJ, Watanabe RM

Broad Institute of Harvard and Massachusetts Institute of Technology, Cambridge, Mass., USA

Nat Genet 2010;42:142-148

Background: Glucose levels $2 \mathrm{~h}$ after an oral glucose challenge are a clinical measure of glucose tolerance used in the diagnosis of T2DM.

Methods: A meta-analysis of 9 genome-wide association studies ( $\mathrm{n}=15,234$ non-diabetic individuals) and a follow-up of 29 independent loci $(\mathrm{n}=6,958-30,620)$.

Results and Conclusion: Variants at the gastric inhibitory polypeptide receptor (GIPR) locus associated with $2 \mathrm{~h}$ glucose level $\left(\mathrm{rs10423928,} \beta(\mathrm{SEM})=0.09(0.01) \mathrm{mmol} / \mathrm{l}\right.$ per A allele, $\left.\mathrm{p}=2.0 \times 10^{-15}\right)$ were identified. The GIPR A-allele carriers also showed decreased insulin secretion $(\mathrm{n}=22,492$; insulinogenic index, $\mathrm{p}=1.0 \times 10^{-17}$; ratio of insulin to glucose area under the curve, $\left.\mathrm{p}=1.3 \times 10^{-16}\right)$ and diminished incretin effect $\left(\mathrm{n}=804 ; \mathrm{p}=4.3 \times 10^{-4}\right)$. Other variants at ADCY5 $\left(\mathrm{rs} 2877716, \mathrm{p}=4.2 \times 10^{-16}\right)$, VPS13C $\left(\mathrm{rs} 17271305, \mathrm{p}=4.1 \times 10^{-8}\right), \operatorname{GCKR}\left(\mathrm{rs} 1260326, \mathrm{p}=7.1 \times 10^{-11}\right)$ and TCF7L2 $(\mathrm{rs} 7903146, \mathrm{p}=4.2 \times$ 
$\left.10^{-10}\right)$ were also associated with $2 \mathrm{~h}$ glucose. Of the three newly implicated loci (GIPR, ADCY5 and VPS13C), only ADCY5 was found to be associated with T2DM in collaborating studies $(\mathrm{n}=35,869$ cases, 89,798 controls, OR $=1.12,95 \%$ CI $\left.1.09-1.15, \mathrm{p}=4.8 \times 10^{-18}\right)$.

The 'incretin effect' denotes the phenomenon by which plasma insulin levels increase more from oral glucose intake than from the same amount of glucose administered intravenously. The incretins are hormones secreted from the gastrointestinal tract into the circulation in response to nutrient ingestion. They potentiate glucose-stimulated insulin secretion from islet $\beta$-cells. Glucose-dependent insulinotropic polypeptide, also known as gastric inhibitory polypeptide (GIP), and glucagon-like peptide-1 (GLP-1), act in an additive manner and are considered responsible for $50-70 \%$ of the postprandial insulin responses. In patients with T2DM, however, their contribution was found to be less than $20 \%$. Interestingly, despite the reduced insulinotropic effect in patients with T2DM, the secretion of GIP appears to be relatively unchanged, suggesting a possible problem with its receptor GIPR.

Genomic-wide association (GWAS) has contributed to the identification of many T2DM-associated loci. In the current study, GWAS revealed 3 new loci associated with 2-h glucose: GIPR (gastric inhibitory polypeptide receptor, rs10423928; VPS13C [vacuolar protein sorting 13 homolog C]) and ADCY5 (adenylate cyclase). Investigations of the latter two loci did not reveal a role in early insulin secretion.

The GIPR variant, the SNP rs10423928 A allele, however, was associated with increased 2-h glucose; a lower insulinogenic index, representing reduction in the early phase of insulin secretion; a lower ratio of insulin to glucose area under the curve, which is an integrated measure of insulin response over the 2-h OGTT, and a lower 2-h insulin level. As expected, GIPR variation was not associated with insulin measures of intravenous glucose tolerance test. Interestingly, deletion of GIPR in an animal model shows mild glucose intolerance and reduced insulin secretion in response to an oral glucose challenge, but normal response to an intraperitoneal glucose challenge. Finally, the rs10423928 A allele was moderately associated with an increased risk of T2D in 19,091 individuals with diabetes, compared with 38,508 individuals without diabetes. These data support the hypothesis that a defect of the GIPR could be part of the T2D pathophysiology [4].

\section{New concerns}

\section{Youth with obesity and obesity-related type 2 diabetes mellitus demonstrate abnormalities in carotid structure and function}

Urbina EM, Kimball TR, McCoy CE, Khoury PR, Daniels SR, Dolan LM

Department of Pediatrics, Cincinnati Children's Hospital and University of Cincinnati, Cincinnati, Ohio, USA

Elaine.Urbina@cchmc.org

Circulation 2009;119:2913-1919

Background: Adults with obesity or T2DM are at higher risk for stroke and myocardial infarction. Increased carotid intima-media thickness (cIMT) and stiffness are associated with these adverse outcomes. The aim of this study was to determine whether similar changes exist in youth with obesity and T2DM.

Methods: Carotid ultrasound for cIMT measurement was performed, as well as measures of stiffness (the Young elastic modulus and $\beta$ stiffness index), anthropometric and laboratory values and blood pressure were measured in 182 lean, 136 obese, and 128 T2DM youth (aged 10-24 years).

Results: Cardiovascular risk factors worsened from lean to obese to T2DM groups. T2DM subjects had greater cIMT than that in lean and obese subjects for the common carotid artery and bulb. For the internal carotid artery, cIMT measurements in both obese and T2DM groups were thicker than in the lean group. The carotid arteries were stiffer in obese and T2DM groups than in the lean group. Determinants of cIMT were group, group $\times$ age interaction, sex, and systolic blood pressure for the common carotid artery $\left(\mathrm{r}^{2}=0.17\right)$; age, race, and systolic blood pressure for the bulb $\left(\mathrm{r}^{2}=0.16\right)$; and age, race, sex, systolic blood pressure, and total cholesterol for the internal carotid artery $\left(\mathrm{r}^{2}=0.21\right)$. Age, systolic blood pressure, and diastolic blood pressure were determinants of all measures of carotid 
stiffness, with sex adding to the Young elastic modulus $\left(r^{2}=0.23\right)$, and body mass index $Z$ score, group, and group $\times$ age interaction contributing to the $\beta$ stiffness index $\left(\mathrm{r}^{2}=0.31\right.$; all $\left.\mathrm{p}<0.0001\right)$.

Conclusions: Youth with obesity and T2DM have abnormalities in carotid thickness and stiffness that should alert healthcare practitioners to address cardiovascular risk factors early to prevent an increase in the incidence of stroke and myocardial infarction.

\section{Influence of duration of diabetes, glycemic control, and traditional cardiovascular risk factors on early atherosclerotic vascular changes in adolescents and young adults with type 2 diabetes mellitus}

Shah AS, Dolan LM, Kimball TR, Gao Z, Khoury PR, Daniels SR, Urbina EM

Cincinnati Children's Hospital Medical Center, Division of Endocrinology, Cincinnati, Ohio, USA

amy.shah@cchmc.org

J Clin Endocrinol Metab 2009;94:3740-1745

Background: Carotid intima-media thickness (IMT) provides a mechanism for detecting early atherosclerosis. Factors that may contribute to early changes in carotid IMT in youth with T2DM were studied. Moreover, predictors of increased carotid IMT were sought.

Methods: Demographic, anthropometric, laboratory data and carotid imaging were obtained in 129 youth of mixed ethnicity, ages 10-23 years.

Results: Carotid IMT increased with higher glycosylated hemoglobin (HbA1c) levels and longer duration of diabetes. Regression modeling showed that HbA1c and duration of diabetes in the presence of traditional cardiovascular risk factors (male sex, LDL cholesterol, and blood pressure) were independent determinants of carotid IMT. Logistic regression analysis demonstrated that each $1 \%$ increase in HbA1c or each year increase in duration of T2DM is associated with approximately $30 \%$ increased odds of a thicker carotid IMT.

Conclusions: Poorer glycemic control and longer disease duration have independent adverse effects on carotid IMT in youth with T2DM. These adverse effects appear to be more prominent in males.

'For the thing which I did fear is come upon me, and that which I was afraid of hath overtaken me' (Job chapter 3, verse 25). One of the main concerns with the new epidemic of 'Diabesity' is the secondary morbidity associated with these disorders, and mainly the appearance of complications early in life, subjecting affected people to many years of morbidity. These two articles describe early atherosclerotic changes in obese and T2DM patients. Although the clinical manifestations of cardiovascular disease appear in adulthood, it is well known that atherosclerosis begins already in childhood. Hyperlipidemia, obesity, hypertension, diabetes and smoking are risk factors for the acceleration of atherosclerosis both in adults and in children. While the development of atherosclerosis in children was initially based on autopsy studies, non-invasive techniques now enable detection of early anatomical and physiological changes. Carotid intima-media thickness (IMT) measurements, which have been used as a surrogate endpoint for the progression and regression of atherosclerotic cardiovascular disease since the 1990s, enable identification of target organ damage. An association between IMT and the presence and severity of atherosclerosis has been documented in adults. In children, IMT was shown to be significantly increased in those with familial hypercholesterolemia, morbid obesity, metabolic syndrome or type 1 diabetes mellitus (T1DM). The study by Urbina et al. is the first to examine the effect of T2DM on IMT among adolescents. Early changes in both vascular structure and function are demonstrated in obese adolescents, and more severely in those with T2DM. Shah et al.'s study investigated factors that may contribute to changes in carotid IMT. Of youth with T2DM, common carotid IMT was elevated in $13.4 \%$, bulb IMT in $16.5 \%$, and internal carotid IMT in $18.9 \%$. These prevalence rates are concerning, as young adults with increased IMT have increased likelihood of myocardial infarction and stroke. Elevated carotid IMT was found to be associated with higher HbA1c concentrations and longer duration of T2DM. In addition, the traditional cardiovascular risk factors, including blood pressure, LDL cholesterol and male sex were also important determinants of carotid IMT in this population. These data establish $\mathrm{HbA} 1 \mathrm{C}$ and duration of diabetes as independent factors in the progressive thickening of carotid IMT. However, there is light in the tunnel. Evidence that improvement in glucose control at an early age may reduce the progression of atherosclerosis should encourage clinicians to aggressive interventions for risk reduction. 


\section{Predictive value of albuminuria in American-Indian youth with or without type 2 diabetes}

Kim NH, Pavkov ME, Knowler WC, Hanson RL, Weil EJ, Curtis JM, Bennett PH, Nelson RG

Diabetes Epidemiology and Clinical Research Section, National Institute of Diabetes and Digestive and Kidney

Diseases, National Institutes of Health, Phoenix, Ariz., USA

Pediatrics 2010;125:e844-851

Objective: To examine the prognostic significance of elevated albuminuria in youth with T2DM.

Methods: Cross-sectional and prospective studies were conducted on Pima Indian youth aged 5-19 years at baseline between 1982 and 2007. Prevalence and sequential changes in the level of microalbuminuria $(30<$ or = albumin-to-creatinine ratio $[\mathrm{ACR}]<300 \mathrm{mg} / \mathrm{g})$ and macroalbuminuria $(\mathrm{ACR}>$ or $=300$ $\mathrm{mg} / \mathrm{g}$ ) and incidence of macroalbuminuria were computed according to the presence or absence of T2DM.

Results: The prevalence of microalbuminuria and macroalbuminuria was 6.5 and $0.6 \%$ in the 3,856 non-diabetic youth and 18.5 and $2.9 \%$ in the 103 youth with diabetes, respectively. 141 of $187(75.4 \%)$ non-diabetic youth, but only 1 of $14(7.1 \%$ ) diabetic youth with an elevated ACR (> or = $30 \mathrm{mg} / \mathrm{g}$ ) regressed to an undetectable or normal ACR $(<30 \mathrm{mg} / \mathrm{g})$ on subsequent examination. In a subset of 2,666 youth with a median follow-up of 8.1 years, 36 non-diabetic and 30 diabetic youth with baseline ACRs of $<300 \mathrm{mg} / \mathrm{g}$ developed macroalbuminuria. For a given ACR, the incidence of macroalbuminuria was 15.9-fold (95\% CI 11.1-22.6) higher in the diabetic than in the non-diabetic youth.

Conclusions: Elevated albuminuria is infrequent and largely transient in non-diabetic youth, but it is relatively frequent and largely persistent in those with diabetes. Microalbuminuria in youth with T2DM strongly predicts progression to macroalbuminuria, which supports annual screening for albuminuria.

The Pima Indians have been called 'pathfinders for health' because of their high level of volunteering to participate in research studies. The cooperative research between the Pima Indians and the $\mathrm{NIH}$, which began in 1963, continues to this day. Since 1965, each member of the population, aged 5 years and older, has been invited to participate in a study investigation every other year. While the overall incidence of diabetes in the Pima Indians has not changed over the past 40 years, the incidence of diabetes among those less than 15 years of age has increased nearly 6 -fold, subsequent to the increasing prevalence and degree of obesity at younger ages [5]. The current prospective study reported a 3 -fold higher incidence of microalbuminuria in youth with T2DM than in those without (18.5 vs. $6.5 \%)$. Importantly, in more than half of the individuals with diabetes, microalbuminuria preceded the diagnosis of T2DM. Microalbuminuria was transient in $75 \%$ of individuals without diabetes, compared to only $7 \%$ of those with diabetes. Microalbuminuria strongly predicted progression to macroalbuminuria, with $27 \%$ of youth with T2DM progressing to macroalbuminuria. The authors concluded that the findings of this population afford examination of the impact of albuminuria in other populations. Of note, although microalbuminuria was initially attributed to diabetes among Canadian First Nation Children, biopsy results demonstrated a non-diabetic renal disease in the form of immune complex diseases or glomerulosclerosis in all cases [6].

\section{New hopes}

\section{Metformin associated with lower cancer mortality in type 2 diabetes: ZODIAC-16}

Landman GW, Kleefstra N, van Hateren KJ, Groenier KH, Gans RO, Bilo HJ

Internal Medicine, Isala Clinics, Zwolle, The Netherlands

g.w.d.landman@isala.nl

Diabetes Care 2010;33:322-326

Background: Several studies have suggested an association between specific diabetes treatment and cancer mortality. The authors studied the association between metformin use and cancer mortality in a prospectively followed cohort. 
Methods: In 1998 and 1999, 1,353 patients with T2DM were enrolled in the Zwolle Outpatient Diabetes project Integrating Available Care (ZODIAC) study in the Netherlands. Vital status was assessed in January 2009. Cancer mortality rate was evaluated using standardized mortality ratios (SMRs).

Results: Median follow-up time was 9.6 years, average age at baseline was 68 years, and average HbA1c was $7.5 \%$. Of the patients, 570 died, of which 122 died of malignancies. The SMR for cancer mortality was 1.47 (95\% CI 1.22-1.76). In patients taking metformin compared with patients not taking metformin at baseline, the adjusted hazard ratio for cancer mortality was 0.43 (95\% CI $0.23-0.80)$, and with every increase of $1 \mathrm{~g}$ of metformin hazard ratio was 0.58 (95\% CI $0.36-0.93$ ).

Conclusions: In general, patients with T2DM are at increased risk for cancer mortality. In this study, metformin use was associated with lower cancer mortality compared with non-use of metformin. Although the design cannot provide a conclusion about causality, data suggest a protective effect of metformin on cancer mortality.

This prospective study showed that use of metformin in patients with diabetes was associated with a lower rate of cancer-related mortality. Increasing evidence from cells, animal models and humans suggests that metformin, a mainstay of T2DM, could be a potent antitumor medicine [7]. In vitro studies with cell cultures demonstrated that metformin benefited human lung, prostate, breast, colon and ovarian cancers. In a recent study in mice, low doses of metformin combined with chemotherapy reduced tumors faster and prolonged remission to a greater degree than did chemotherapy alone. Most importantly, the mechanism involved targeting cancer stem cells, which appear to resist conventional chemotherapies. In humans, epidemiological studies reported treatment with metformin to be associated with a lower overall risk for cancer than without treatment, or than treatment with insulin or antidiabetic drugs. Patients with breast cancer who were treated with both neoadjuvant chemotherapy and metformin had a higher response rate than those treated with other diabetes medications. Finally, as presented in the current article, in patients with diabetes, metformin use was associated with lower cancer mortality. It has been suggested that metformin may influence cancer cells indirectly through insulin-mediated effects, or it may directly affect cell proliferation and apoptosis of cancer cells. Pediatricians need to follow these data closely. Recent reports suggest a relationship between childhood obesity, and an increased risk of adult pancreatic and colon cancer. Adults with T2DM are at increased risk to develop cancer. Thus, if indeed metformin treatment will be found to have antitumorgenic effect, it will be essential for children and youth with T2DM.

\section{Screening for type 2 diabetes in obese youth}

Shah S, Kublaoui BM, Oden JD, White PC

Department of Pediatrics, University of Texas Southwestern Medical Center, Dallas, Tex., USA

Pediatrics 2009;124:573-579

Objective: To assess available blood tests as potential screening tools for impaired glucose tolerance (IGT) and T2DM.

Methods: 468 obese (mean BMI $>34.4 \mathrm{~kg} / \mathrm{m}^{2}$ ) children, including a subgroup with serum fasting insulin levels of $>15 \mu \mathrm{IU} / \mathrm{ml}$, were studied. An oral glucose tolerance test was performed on each patient, fasting and 2-hour post-load serum glucose and insulin levels were obtained as well as HbA1c, and 1,5-anhydroglucitol (insulin-resistant subgroup only) levels. All values and calculated HOMA-IR were used as predictors for exceeding various 2 -hour BG cut-offs.

Results: In the insulin-resistant subgroup, $3(2 \%)$ patients had T2DM and $23(12 \%)$ had IGT. Optimal sensitivity and specificity to detect T2DM were respectively 99 and $96 \%$ at $\mathrm{HbA} 1 \mathrm{c}>$ or $=6.0 \%$, and 96 and $88 \%$ at 1,5 -anhydroglucitol $<17.0 \mu \mathrm{g} / \mathrm{ml}$, with lower values for fasting BG and the HOMA-IR. In the entire study group, $9(2 \%)$ patients had T2DM and $44(9 \%)$ had IGT. Optimal sensitivity and specificity to detect T2DM were respectively 86 and $85 \%$ at HbA1c levels of 5.7, 88, and $93 \%$ at a fasting BG level of $104 \mathrm{mg} / \mathrm{dl}$, and 62 and $70 \%$ at an HOMA-IR of 7.9 .

Conclusions: HbA1c, 1,5-anhydroglucitol, and fasting BG levels are good predictors of T2DM in obese children, whereas HOMA-IR values are not. HbA1c and 1,5-anhydroglucitol are excellent predictors of T2DM in insulin-resistant obese children. 
The escalating epidemic of childhood obesity calls for a simple diagnostic tool to identify those with impaired glucose tolerance and those with undiagnosed T2DM. Performing an oral glucose tolerance test (OGTT) on all obese children is a cumbersome task. Indeed, as the authors of the current study point out, many clinicians fail to follow the ADA guidelines for performing OGTT in obese children. Moreover, screening tests need be repeated periodically, especially when children continue to gain weight, and even when their baseline results are normal. The aim of this study was to investigate the possible utilization of fasting glucose, $\mathrm{HbA} 1 \mathrm{c}, \mathrm{HOMA}-\mathrm{IR}$ and 1,5-anhydroglucitol as simpler screening tests. 1,5-Anhydroglucitol, also known as 1,5-AG, is a naturally occurring monosaccharide found in nearly all foods. Since hardly any of 1,5-AG is metabolized, its level remains relatively constant in the blood and tissues. Normally, a small amount of 1,5-AG, equal to the amount ingested, is excreted in the urine. When blood glucose exceeds $180 \mathrm{mg} / \mathrm{dl}$, and glycosuria occurs, competitive inhibition blocks 1,5-AG from being reabsorbed, and its blood level decreases. Thus, low levels of 1,5-AG indicate hyperglycemia. Moreover, in subjects with diabetes, this assay indicates excessive glycemic variability, even in the presence of relatively low HbA1c levels.

In the current study, the authors found 1,5-AG levels to only modestly predict impaired glucose tolerance among children. However, HbA1c, 1,5-AG, and fasting BG levels were found to be easily implemented screening tests for T2DM in obese children.

\section{Food for thought}

\section{Ethnic differences in triglyceride levels and high-density lipoprotein lead to underdiagnosis of the metabolic syndrome in black children and adults}

\section{Sumner AE}

Clinical Endocrinology Branch, NIDDK, NIH, Bethesda, Md., USA

Annes@intra.niddk.nih.gov

J Pediatr 2009;155:S7 e7-11

Background: The metabolic syndrome (MetS) was designed to identify individuals at high risk for the T2DM and cardiovascular disease. Compared with whites, blacks have higher rates of diabetes and cardiovascular disease. Paradoxically, blacks have a lower prevalence of the MetS. According to the criteria set by National Cholesterol Education Treatment Program-Adult Treatment Panel III, to diagnose the MetS, 3 of 5 characteristics must be present. These characteristics are low high-density lipoprotein levels, increased triglyceride levels, central obesity, hypertension, and fasting hyperglycemia. Examining each of these factors individually, blacks are more likely than whites to have obesity, hypertension, and diabetes. In contrast, blacks are less likely than whites to have either elevated triglyceride or low high-density lipoprotein levels. Ethnic differences in lipid levels may largely explain why blacks have a lower than expected prevalence of the MetS. Children and adults ethnic differences in the epidemiologic study of conditions associated with the MetS, as well as focus on each of the parameters of the MetS are reviewed.

Conclusions: An ethnic-specific formulation of the lipid criteria in the MetS may lead to better identification of blacks at high risk for development of diabetes and cardiovascular disease.

The metabolic syndrome (MetS) comprises the clustering of risk factors that identify individuals at increased risk of CVD and diabetes. Basically, it involves the co-occurrence of at least three of the following: obesity (defined either by BMI or waist circumference), dyslipidemia including elevated TG and/or low HDL, hypertension and abnormal glucose metabolism (fasting glucose, impaired glucose tolerance). In children and in youth, the metabolic syndrome may be a particularly important tool to predict and prevent adult diseases. Between 1995 and 2000, about 10 papers regarding the metabolic syndrome in children were published, compared to around 300-400 articles per year over the last 3 years. However, the concept of the metabolic syndrome in children and adolescents has undergone long and painful labor, and the newborn still has many developmental disorders. First, several definitions were proposed by the WHO, the National Cholesterol Education Program Adult Treatment Panel III (ATP III) [5], the European Group for the Study of Insulin Resistance, and the International 
Diabetes Federation (IDF). The lack of unified criteria makes it difficult to compare prevalence rates between populations and at-risk groups. In children, the determination of cut-off points is even more complicated than in adults, as age must also be considered.

Sumner's recent article is one of several that elucidate problems in defining the metabolic syndrome. She points out that while obesity, insulin resistance, diabetes and hypertension are much more prevalent in blacks than in whites, the prevalence of the metabolic syndrome is lower in blacks. It is suggested that the factor of dyslipidemia has a disproportionate effect on metabolic syndrome diagnosis, as high triglyceride and low HDL levels occur together, due to their reciprocal clearance from blood circulation. These data may be relevant to other ethnic populations, with implications on the predictive value of the metabolic syndrome for future morbidity. Moreover, considering established risk factors that are not included in the metabolic syndrome, such as birth weight, family history, physical activity, age of puberty, and LDL cholesterol level, it seems that we might need other tools for risk prediction.

\section{Childhood obesity, other cardiovascular risk factors, and premature death}

Franks PW, Hanson RL, Knowler WC, Sievers ML, Bennett PH, Looker HC

Diabetes Epidemiology and Clinical Research Section, National Institute of Diabetes and Digestive and Kidney

Diseases, National Institutes of Health, Phoenix, Ariz., USA

paul.franks@medicin.umu.se

N Engl J Med 2010;362:485-493

Background: The effect of childhood risk factors for cardiovascular disease on adult mortality is poorly understood.

Methods: A cohort of 4,857 American-Indian children without diabetes (mean age 11.3 years), who were born between 1945 and 1984, were studied to assess whether BMI, glucose tolerance, blood pressure and cholesterol levels predicted premature death. Risk factors were standardized according to sex and age.

Results: There were 166 deaths from endogenous causes (3.4\% of the cohort) during a median follow-up period of 23.9 years. Rates of death from endogenous causes among children in the highest quartile of BMI were more than double those among children in the lowest BMI quartile (incidence-rate ratio 2.30; 95\% CI 1.46-3.62). Rates of death from endogenous causes among children in the highest quartile of glucose intolerance were $73 \%$ higher than those among children in the lowest quartile (incidence-rate ratio $1.73 ; 95 \%$ CI 1.09-2.74). No significant associations were seen between rates of death from endogenous or external causes and childhood cholesterol levels or systolic or diastolic blood-pressure levels on a continuous scale, although childhood hypertension was significantly associated with premature death from endogenous causes (incidence-rate ratio 1.57; 95\% CI 1.10-2.24).

Conclusions: Obesity, glucose intolerance, and hypertension in childhood were strongly associated with increased rates of premature death from endogenous causes in this population. In contrast, childhood hypercholesterolemia was not a major predictor of premature death from endogenous causes.

The opening sentence of this article is overwhelming: 'Despite recent increases in life expectancy, the rising global prevalence of obesity may reverse this trend...'. The study assessed BMI, glucose tolerance, blood pressure and cholesterol levels in 4,857 American-Indian children and adolescents (aged 5-20 years) without diabetes. During a 24-year follow-up, 3.4\% died prematurely (before age 55 years) from endogenous causes, i.e. by disease or self-inflicted injury. Of these, alcoholic liver disease accounted for the majority (59\%), followed by cardiovascular disease $(22 \%)$, infections $(21 \%)$, cancer $(12 \%)$, diabetes or diabetic nephropathy $(10 \%)$ and acute alcoholic poisoning or drug overdose $(9 \%)$. 33 deaths were attributed to other causes. High BMI, glucose intolerance and hypertension in childhood were strongly associated with increased rates of premature death from endogenous causes, whereas hypercholesterolemia was not. Thus childhood obesity predicted premature death.

The mirror image of obesity and premature death is interesting. In rodent models, longevity has been associated with caloric restriction. Caloric restriction has been shown to extend lifespan through induction of sirtuins. Sirtuins are a family of seven proteins, SIRT1-SIRT7. Sirtuin 1 (SIRT1) is the mammalian homologue of the evolutionarily conserved silent information regulator 2 (SIR2), a deacetylase that regulates lifespan in response to caloric restriction in many organisms. In a combined in vivo and in vitro study, gene and protein expression of SIRT1 were significantly reduced in relation to insulin resistance and MetS [8]. SIRT1 was downregulated in individuals with prediabetes compared 
to those with normal glucose metabolism. Decreased SIRT1 was associated with a shorter lifespan. These data suggest that SIRT1 might be a new therapeutic target for the prevention of disease related to insulin resistance. It is therefore not surprising that in 2008 , GlaxoSmithKline bought the company who developed a small molecule drug aimed at increasing SIRT1 activity as an 'anti-aging drug'. I wait eagerly, not only to realize the promise of longevity, but to be skinny as well.

\section{Concepts revised}

\section{Heritability of childhood weight gain from birth and risk markers for adult metabolic disease in prepubertal twins}

Beardsall K, Ong KK, Murphy N, Ahmed ML, Zhao JH, Peeters MW, Dunger DB

Department of Paediatrics, University of Cambridge, Addenbrooke's Hospital, Cambridge, UK

J Clin Endocrinol Metab 2009;94:3708-3713

Objective: Associations between size at birth, postnatal weight gain, and potential risk for adult disease have been variably explained by in utero exposures or genetic risk that could affect both outcomes. A twin model to explore these hypotheses was utilized.

Methods: 100 pairs of healthy twins aged 8.9 years (range 7.2-10.9) had fasting blood samples collected, blood pressure (BP) measured, and anthropometry assessed.

Results: Mean birth weights in both monozygotic and dizygotic twins were -0.90 SDS lower than the UK reference. In postnatal life, $58 \%$ of monozygotic twins and 59\% of dizygotic twins showed rapid weight gain (a change of more than +0.67 in weight SDS) from birth. Postnatal weight gain was positively associated with sum of skinfolds $(\mathrm{r}=0.51 ; \mathrm{p}<0.0005)$, fasting insulin levels $(\mathrm{r}=0.35 ; \mathrm{p}<0.0005)$, systolic BP $(\mathrm{r}=0.30 ; \mathrm{p}<0.0005)$, and diastolic BP $(\mathrm{r}=0.15 ; \mathrm{p}<0.05)$ at follow-up. Heritability estimates (additive genetic components) were calculated using variance components models for: birth weight, $44 \%$; postnatal weight gain, $80 \%$; childhood height, $89 \%$; body mass index, $72 \%$; sum of skinfolds, $89 \%$; waist circumference, $74 \%$; fasting insulin, $65 \%$; systolic BP, $33 \%$, and diastolic BP, $29 \%$.

Conclusions: Postnatal weight gain from birth, rather than birth weight, was associated with childhood risk markers for adult metabolic disease. Childhood weight gain was highly heritable, and genetic factors associated with postnatal weight gain are likely to also contribute to risks for adult disease.

\section{Timing and tempo of first-year rapid growth in relation to cardiovascular and metabolic risk profile in early adulthood}

Leunissen RW, Kerkhof GF, Stijnen T, Hokken-Koelega A

Department of Pediatrics, Subdivision of Endocrinology, Erasmus Medical Center/Sophia Children's Hospital, Rotterdam, The Netherlands

r.leunissen@erasmusmc.nl

JAMA 2009;301:2234-2242

Background: Growth during infancy appears to be an important determinant of cardiovascular disease and type 2 diabetes later in life. To specify which period in the first year of life is related to determinants of cardiovascular disease and T2DM in early adulthood and to investigate the association between tempo of first-year weight gain ( $>0.67$ SDs) and these determinants.

Methods: Observational study using longitudinal data collected in the Programming Factors for Growth and Metabolism (PROGRAM) study of 217 healthy participants, aged 18-24 years, including a relatively large sample of participants born small for gestational age and participants with short stature. The association of cardiovascular disease and T2DM with tempo of weight gain was assessed in a subgroup of 87 participants.

Results: Weight gain in the first 3 months of life was inversely associated with insulin sensitivity $(\beta$ $-0.223 ; 95 \% \mathrm{CI}-0.386$ to -0.060$)$ and serum high-density lipoprotein cholesterol level $(\beta-0.053 ; 95 \%$ CI -0.090 to -0.016$)$ and positively associated with waist circumference ( $\beta$ 1.437; 95\% CI $0.066-$ 
2.808 ), acute insulin response ( $\beta 0.210 ; 95 \%$ CI $0.024-0.395)$, ratio of total cholesterol to high-density lipoprotein cholesterol ( $\beta$ 0.052; 95\% CI 0.010-0.094), and level of triglycerides $(\beta 0.066 ; 95 \%$ CI 0.003-0.129) in early adulthood. Rapid weight gain during the first 3 months of life resulted in a higher percentage of body fat, more central adiposity, and reduced insulin sensitivity in early adulthood than when slower weight gain occurred during the entire first year.

Conclusion: Rapid weight gain in the first 3 months of life is associated with several determinants of cardiovascular disease and T2DM in early adulthood.

Since the introduction of Barker's hypothesis regarding the developmental origins of adult diseases, many epidemiological studies have reported a strong association between growth retardation in uterus and long-term adverse effects such as T2DM, coronary heart disease, hypertension and stroke. The above two articles show the period of postnatal catch-up growth, and not just fetal life, to be an important determinant of adult disease risk.

In the first article, Beardsall et al. used models of monozygotic (MZ) and dizygotic (DZ) twins to explore the contribution of postnatal weight gain to markers of adult metabolic disease states, including adiposity, arterial blood pressure and fasting insulin levels. This elegant model enables estimation of the contribution of fetal genotype, maternal environment and postnatal weight gain. Both MZ and DZ twins, who were growth restricted at birth, showed catch-up growth in both weight and height by ages 7-11 years. Neither birth weight nor discordance between twin pairs was associated with markers of metabolic disease. Outcomes were strongly related to postnatal weight gain. The heritability of postnatal weight gain and fasting insulin were assessed at 80 and $65 \%$ respectively. While Beardsall et al.'s study did not investigate the exact timing of catch-up growth, Leunissen et al.'s study found that in the first 3 months of life, an increased gain in weight, relative to height, was associated with all the important determinants of the metabolic syndrome, specifically: increased waist circumference, low insulin sensitivity, low HDL and increased triglyceride levels in early adulthood. Moreover, the tempo of weight gain was also found important. Children with rapid weight gain within the first 3 months had higher percentages of body fat, central adiposity and reduced insulin sensitivity later. Although nutritional data was not available, the use of nutrient-enriched formulas may be one explanation for rapid weight gain. Anxious to see their 'malnourished baby' achieving normal weight percentiles as soon as possible, parents often implement aggressive dietary management with considerable caloric intake supplementation. In light of these findings, we should be cautious in our aims for rapid catch-up.

\section{Prolonged juvenile states and delay of cardiovascular and metabolic risk factors: the Fels Longitudinal Study}

Sun SS, Schubert CM

Department of Biostatistics, School of Medicine, Virginia Commonwealth University, Richmond, Va., USA

stephen_cook@urmc.rochester.edu

J Pediatr 2009;155:S7 e1-6

Background: The influence of a prolonged juvenile state on the onset of the metabolic syndrome, cardiovascular disease, and T2DM later in life was studied.

Methods: Prolongation of a juvenile state as a retarded tempo of growth was determined by the timing of peak height velocity in each subject and relate the retarded tempo of growth to metabolic syndrome, cardiovascular disease, and T2DM later in life by use of serial data of 237 study participants (119 men and 118 women) enrolled in the Fels Longitudinal Study.

Results: Children who matured early tended to have greater body mass index, waist circumference, and percent of body fat and were more likely to have adverse cardiovascular risk profiles than children who matured late. The differences in these risk factors between early and late maturers were significant for percent body fat, fasting plasma triglycerides, and fasting plasma insulin.

Conclusions: The analyses disclosed a clear separation between early and late maturers in the appearance of these risk factors in young adulthood.

This study demonstrates the value and power of longitudinal studies in revealing predictors of certain conditions. The Fels Longitudinal Study began in 1929, after the president of Antioch College in Yellow Springs, Ohio, posed the question, 'What makes people different?' He believed that a longi- 
tudinal study from birth to adulthood would be required to answer this question. Samuel Fels, a Philadelphia businessman and philanthropist, established the Fels Research Institute. Data measured by the Fels Longitudinal Study has since become the core of numerous works. The current study is the first to elucidate the effects of maturation rate on metabolic variables and risk factors for cardiovascular disease over 40 years of lifespan. It is known that overweight children, particularly those of certain minority groups, those born small for gestational age, females with PCOS, and children with family histories of T2DM, are at risk for developing the MetS. Early maturation is now shown to be another risk factor for developing the MetS. Children who matured early tended to have greater BMI and waist circumference, a higher percentage of body fat, and elevated levels of fasting plasma triglycerides and fasting plasma insulin in adulthood compared with late maturers. As such, a new risk group for development of the MetS has been identified, for which careful follow-up is needed.

\title{
A novel, non-invasive ${ }^{13} \mathrm{C}$-glucose breath test to estimate insulin resistance in obese prepubertal children
}

\author{
Jetha MM, Nzekwu U, Lewanczuk RZ, Ball GD \\ Department of Pediatrics, Faculty of Medicine and Dentistry, University of Alberta, Edmonton, Alta., Canada \\ J Pediatr Endocrinol Metab 2009;22:1051-1059
}

Background: Insulin resistance (IR) is an important risk factor for the development of T2DM in obese boys and girls. The objective of this cross-sectional study of obese prepubertal children $(\mathrm{n}=39)$ was to compare estimates of IR using a novel, non-invasive technique $\left({ }^{13} \mathrm{C}\right.$-glucose breath test) with common indices of IR derived from an oral glucose tolerance test (OGTT).

Methods: For the ${ }^{13} \mathrm{C}$-glucose breath test, samples were collected before and $90 \mathrm{~min}$ after ingestion of 25 mg ${ }^{13} \mathrm{C}$-labeled glucose. For the OGTT, glucose and insulin samples were collected at $0,15,30,45,60$, 90 and $120 \mathrm{~min}$. The homeostatic model assessment of insulin resistance (HOMA-IR), quantitative insulin sensitivity check index (QUICKI), insulin area-under-the-curve (AUC), and sum-of-insulin were calculated as indices of IR.

Results: A significant, but moderate, associations between the ${ }^{13} \mathrm{C}$-glucose breath test and fasting insulin $(\mathrm{r}=-0.50 ; \mathrm{p}<0.01), 2$-hour insulin $(\mathrm{r}=-0.40 ; \mathrm{p}<0.05)$, HOMA-IR $(\mathrm{r}=-0.51 ; \mathrm{p}<0.01)$, QUICKI $(\mathrm{r}=$ $0.53 ; \mathrm{p}<0.01)$, insulin AUC ( $\mathrm{r}=-0.22$; NS), and sum-of-insulin $(\mathrm{r}=-0.48 ; \mathrm{p}<0.05)$.

Conclusions: In obese prepubertal children, the ${ }^{13} \mathrm{C}$-glucose breath test can provide a proxy estimate of IR when gold-standard techniques are either unavailable or impractical.

In healthy people, ingested glucose is metabolized in the presence of insulin, producing $\mathrm{CO}_{2}$, which is eliminated by the lungs through expiration. Since glucose can be labeled with a stable isotope tracer $\left({ }^{13} \mathrm{C}\right)$, its metabolism to $\mathrm{CO}_{2}$ can be determined quantitatively. Among subjects with insulin resistance, glucose uptake is impaired and less $\mathrm{CO}_{2}$ is produced. Among adults, the ${ }^{13} \mathrm{C}$-glucose breath test was found to be highly correlated $(r \geq 0.69)$ with IR determined by a hyperinsulinemiceuglycemic clamp [9].

In the current study, estimates of IR generated by the ${ }^{13} \mathrm{C}$-glucose breath test were compared to indices derived from OGTT in prepubertal obese children. A significant, though moderate, concordance was found between ${ }^{13} \mathrm{CO}_{2}$ in breath and fasting insulin, 2-hour insulin, and calculated estimates of insulin resistance such as HOMA and QUICKI. This may be useful, since clinicians often avoid testing children when repeated blood tests are necessary, as in the OGTT. Such an easy to perform, noninvasive test, applicable in both private clinical and non-clinical settings, should be investigated in different populations, including pubertal children, and children of different ethnicities. So far, it seems a promising and practical means of assessing insulin resistance in children. 


\section{Effect of injectable and oral contraceptives on serum lipids}

Berenson AB, Rahman M, Wilkinson $G$

Department of Obstetrics and Gynecology, The Center for Interdisciplinary Research in Women's Health,

The University of Texas Medical Branch, Galveston, Tex., USA

abberens@utmb.edu

Obstet Gynecol 2009;114:786-794

Objective: To estimate the effects of using depot medroxyprogesterone acetate (DMPA) or oral contraceptives (OCs) containing $20 \mu \mathrm{g}$ ethinyl estradiol and $0.15 \mathrm{mg}$ desogestrel on serum lipid levels.

Methods: Serum lipids were measured at baseline and every 6 months thereafter for 3 years in 703 white, African-American, and Hispanic women using DMPA, OC, or non-hormonal birth control. Those who discontinued DMPA were followed for up to 2 additional years. Participants completed questionnaires containing demographic and behavioral measures every 6 months and underwent 24-hour dietary recalls annually. Mixed-model regression analyses and general-estimating-equations procedures were used to estimate changes over time in lipids by method along with their predictors.

Results: Users of OCs experienced significantly greater increases in levels of triglycerides, total cholesterol, very-low-density lipoprotein (VLDL) cholesterol, and high-density lipoprotein (HDL) cholesterol than did non-hormonal-contraceptive users $(\mathrm{p}<0.001)$. However, no difference was noted in the lowdensity lipoprotein (LDL) cholesterol:HDL ratio between OC users and non-hormonal-contraceptive users. Among DMPA users, HDL levels initially decreased for 6 months but then returned to baseline. The LDL:HDL ratio rose in the first 6 months of DMPA use but then dropped back to baseline over the next 24 months. After DMPA was discontinued, triglyceride, VLDL, and HDL levels were significantly higher in women who used OCs than in those who chose non-hormonal $(\mathrm{p}<0.05)$ methods.

Conclusions: Use of very-low-dose OCs containing desogestrel can elevate lipid levels. Users of DMPA were at increased risk of developing an abnormally low HDL level as well as an abnormally high LDL level and an increase in the LDL:HDL cholesterol ratio, although these effects appeared to be temporary.

In the year 2010 we mark the 50th anniversary of the approval of oral contraception (OC). The enormous social impact of the pill is without question [10]. It was key to women's gaining control over their fertility, and an 'equalizer' that afforded women the same sexual freedom that men had traditionally enjoyed. This revolutionary degree of autonomy, equality and freedom led to women's modern economic role. The UN estimates that more than 100 million women worldwide take some form of hormonal contraception. The most serious risks associated with OC use include blood clots, pulmonary embolism, stroke, heart attacks and breast cancer. The current article aimed to resolve controversies on the effect of OC on lipid levels, specifically those of both depot medroxyprogesterone acetate (DMPA) and of the third-generation OC containing only $20 \mu \mathrm{g}$, ethinyl estradiol and $0.15 \mathrm{mg}$ desogestrel. The strength of the study is the long-term follow-up: over 3 years of contraceptive use.

During the study period, OC users experienced statistically significant increases in levels of triglycerides, total cholesterol, VLDL and HDL cholesterol, greater than those experienced by non-hormonal contraceptive users. In fact, OC users were 3-fold more likely to have triglycerides level $>170 \mathrm{mg} / \mathrm{dl}$, and 4-fold more likely to have LDL $>160 \mathrm{mg} / \mathrm{dl}$. The net effect of OC on lipid levels seems to depend on the balance between increased HDL cholesterol and increased triglycerides. DMPA users were 2.5 times more likely than non-hormonal contraceptive users to have a level of $\mathrm{HDL} \leq 35 \mathrm{mg} / \mathrm{dl}$, and almost 3-fold more likely to have abnormal LDL levels. These findings highlight the importance of monitoring lipid profiles in adolescents treated with OC. Of particular concern is the use of OC by adolescents with PCOS who tend to have dyslipidemia as part of their metabolic syndrome. 


\title{
Relationships between 25-hydroxyvitamin D levels and plasma glucose and lipid levels in pediatric outpatients
}

\author{
Johnson MD, Nader NS, Weaver AL, Singh R, Kumar S
}

Department of Pediatric and Adolescent Medicine, Mayo Clinic College of Medicine, Rochester, Minn., USA J Pediatr 2010;156:444-449

Objective: To study the relationships between serum vitamin D levels and plasma glucose or lipid levels in children and adolescents.

Methods: This is a retrospective record review of pediatric outpatients (age 2-18 years) with simultaneous measurement of 25-hydroxyvitamin D $(25[\mathrm{OH}] \mathrm{D})$ and fasting plasma glucose $(\mathrm{n}=302)$ or $25(\mathrm{OH})$ $\mathrm{D}$ and a lipid panel $(\mathrm{n}=177)$. Pearson correlation coefficient was used to estimate the correlation between $25(\mathrm{OH}) \mathrm{D}$ and logarithmic transformed plasma glucose or lipid levels. Plasma glucose and lipid levels were compared in subjects with $25(\mathrm{OH}) \mathrm{D}$ concentrations greater or less than $30 \mathrm{ng} / \mathrm{ml}$.

Results: $25(\mathrm{OH}) \mathrm{D}$ levels were inversely correlated with fasting plasma glucose levels $(\mathrm{r}=-0.20, \mathrm{p}<$ $0.001)$. Lower $25(\mathrm{OH}) \mathrm{D}$ levels were also associated with lower serum high-density lipoprotein cholesterol $(\mathrm{HDL})$ concentrations $(\mathrm{r}=0.41 ; \mathrm{p}<$ or $=0.001)$. The relationship between $25(\mathrm{OH}) \mathrm{D}$ levels and fasting glucose and HDL levels did not vary significantly with sex, age, body mass index Z-score, or season. Children who were vitamin D insufficient $(25(\mathrm{OH}) \mathrm{D}<$ or $=30 \mathrm{ng} / \mathrm{ml})$ had higher fasting plasma glucose $(\mathrm{p}=0.002)$ and lower HDL levels $(\mathrm{p}<0.001)$ than children who were vitamin $\mathrm{D}$ sufficient $(25(\mathrm{OH}) \mathrm{D}>30 \mathrm{ng} / \mathrm{ml})$.

Conclusions: Low 25(OH)D levels in children and adolescents are associated with higher plasma glucose and lower HDL concentrations.

The discovery of the critical roles of vitamin $D$ for overall health is a fascinating story in the history of medicine. First are its osseous effects and the association to rickets. Then came the discovery of its anti-infectious role, from the breakthrough by Niels Finsen, earning him a Nobel Prize in 1903 for the use of a form of 'concentrated light radiation' to treat tuberculosis skin lesions, through the sanatoriums built to treat patients with sunbathing, until the discovery of cathelicidin - an antimicrobial peptide, regulated by vitamin $D$, that serves a critical role in mammalian innate immune defense against invasive bacterial infection. The editorial by Chesney [11] is highly recommended. No wonder that common wisdom says, 'Where the sun is, the doctor ain't'. Recent studies indicate that the sunlight-generated hormone, vitamin $D$, with its plethora of biological effects on diverse tissues, sustains health throughout the body. It is now believed that vitamin $\mathrm{D}$ can protect against multiple sclerosis, T1DM and cancer. Among adults, low levels of vitamin D have been shown to be associated with increased risks of obesity, hypertension, glucose intolerance, T2DM and cardiovascular disease [12]. In the above study the authors examined the association between vitamin D, glucose and lipid levels, as features of the metabolic syndrome. Decreased levels of vitamin D were significantly associated with increasing levels of glucose. While no correlations were found between vitamin $D$ levels and total serum cholesterol, triglyceride and non-HDL, low vitamin levels were associated with low HDL levels. These relationships persisted independent of adiposity, age and sex. Relatively few foods naturally contain vitamin D, the most abundant being oily fish like salmon, sardines, mackerel, and cod liver oil, which are not often consumed by children. In 2008 the American Academy of Pediatrics Committee on Nutrition recommended a minimum daily intake of $400 \mathrm{IU}$ vitamin D for all infants and children, including adolescents. Currently, the most important question is if supplementation will prevent cardiovascular events. One systemic review found only weak evidence of the benefit from vitamin $D$ supplementation [13], and another found no evidence [14]. Currently available data preclude definitive conclusion. We await the results of the National Institutes of Health sponsored trial, VITAL (VITamin D and OmegA-3 TriaL), which will study vitamin D (2,000 IU) and $\omega$-3-fatty acid supplementation, and their effects on heart disease, stroke and cancer. 


\title{
Efficacy and safety of rosuvastatin therapy for children with familial hypercholesterolemia
}

\author{
Avis HJ, Hutten BA, Gagne C, Langslet G, McCrindle BW, Wiegman A, Hsia J, Kastelein JJ, Stein EA \\ Department of Vascular Medicine, Academic Medical Center, University of Amsterdam, Amsterdam, The Netherlands
} J Am Coll Cardiol 2010;55:1121-1126

Background: The efficacy and safety of rosuvastatin therapy for children with familial hypercholesterolemia was studied. In children, statins have been shown to be effective in reducing LDL-C, restoring flow-mediated dilation, and slowing carotid intima-media thickening. However, few children in these trials achieved current LDL-C goals.

Methods: This study comprised a 12-week double-blind, randomized, placebo-controlled trial, followed by a 40-week open-label, titration-to-goal extension phase in 177 pubertal children, ages 10-17 years, with familial hypercholesterolemia. Participants were randomly assigned to placebo or rosuvastatin 5, 10 , or $20 \mathrm{mg}$ once daily.

Results: Compared with placebo, rosuvastatin 5, 10, and $20 \mathrm{mg}$ reduced LDL-C by 38, 45, and 50\%, respectively ( $\mathrm{p}<0.001$ for each group vs. placebo). With a maximum allowed dose of $20 \mathrm{mg}, 40 \%$ achieved the treatment goal of $<110 \mathrm{mg} / \mathrm{dl}$ during the open-label, titration-to-goal phase. Rosuvastatin was well tolerated, with no apparent adverse impact on growth or development.

Conclusions: In children with familial hypercholesterolemia, rosuvastatin $20 \mathrm{mg}$ daily reduced LDL-C by $50 \%$. Nonetheless, only $40 \%$ attained the consensus LDL-C target of $<110 \mathrm{mg} / \mathrm{dl}$, reflecting these patients' high baseline LDL-C levels (mean $232 \mathrm{mg} / \mathrm{dl}$ ).

Rosuvastatin is the highest potency statin presently on the market. It is a competitive inhibitor of the enzyme HMG-CoA reductase, which is the rate-limiting enzyme of cholesterol synthesis. Inhibition of this HMG-CoA reductase in the liver decreases cholesterol synthesis, while increasing synthesis of LDL receptors, thus resulting in increased clearance of low-density lipoprotein (LDL) from the bloodstream. Rosuvastatin was first launched in 2003, however its capture of the market has followed a rough course. Though the drug was marketed as a 'super-statin', its introduction to clinical practice in the absence of reliable data about efficacy and safety was sharply criticized. Nonetheless, the FDA refused to withdraw Resuvastatin from the market. For a happy ending, in 2008 the JUPITER (Justification for the Use of Statins in Primary Prevention: an Intervention Trial Evaluating Rosuvastatin) trial was stopped early due to unequivocal evidence from an independent Data and Safety Monitoring Board of reduced cardiovascular morbidity and mortality in patients treated with rosuvastatin compared with placebo. As much as $50-75 \%$ of drugs used in pediatric medicine have not been studied adequately to provide appropriate labeling information. In 1997, the American Congress passed the FDA Modernization Act, which encouraged pediatric drug development by providing an incentive in the form of additional marketing exclusivity. The consequent increase in pediatric clinical studies has resulted in improved understanding of the pharmacokinetics of drugs prescribed in pediatric medicine, important dose changes, and improved safety for children taking certain drugs [15]. For rosuvastatin, as with all statins, the risk of rhabdomyolysis, and other undesired side effects, be they hepatic, skeletal or renal is a concern. This study is the first to evaluate the efficacy and safety of rosuvastatin in children aged 10-17 years. Its importance therefore lies in testing doses, compliance and safety profile.

\section{Colesevelam hydrochloride: efficacy and safety in pediatric subjects with heterozygous familial hypercholesterolemia}

Stein EA, Marais AD, Szamosi T, Raal FJ, Schurr D, Urbina EM, Hopkins PN, Karki S, Xu J, Misir S, Melino M Metabolic and Atherosclerosis Research Center, Cincinnati, Ohio, USA

ESteinMRL@aol.com

J Pediatr 2010;156:231-236 e1-3

Objective: Efficacy and safety of colesevelam hydrochloride were evaluated in children with heterozygous familial hypercholesterolemia (heFH). 
Methods: This was a randomized, double-blind, multicenter site study in 194 boys and postmenarchal girls aged 10-17 years with heFH (statin-naive or on a stable statin regimen). After a 4-week stabilization period (period I), subjects were randomized to placebo $(\mathrm{n}=65)$, colesevelam $1.875 \mathrm{~g} /$ day $(\mathrm{n}=65)$, or colesevelam $3.75 \mathrm{~g} /$ day $(\mathrm{n}=64)$ for 8 weeks (period II). All patients then received open-label colesevelam $3.75 \mathrm{~g} /$ day for 18 weeks (period III).

Results: At week 8, a significant difference from baseline in LDL cholesterol was reported with colesevelam $1.875 \mathrm{~g} /$ day $(-6.3 \% ; \mathrm{p}=0.031)$ and colesevelam $3.75 \mathrm{~g} /$ day $(-12.5 \% ; \mathrm{p}<0.001)$ compared with placebo. Significant treatment effects were also reported for total cholesterol $(-7.4 \%)$, non-HDL cholesterol $(-10.9 \%)$, HDL cholesterol $(+6.1 \%)$, apolipoprotein A-I $(+6.9 \%)$, and apolipoprotein B $(-8.3 \%)$ and a non-significant effect for triglycerides $(+5.1 \%)$ with colesevelam $3.75 \mathrm{~g} /$ day compared with placebo at week 8 . These treatment effects were maintained during period III.

Conclusions: Colesevelam significantly lowered LDL cholesterol levels in children with heFH.

In October 2009 the FDA approved colesevelam HCl tablets and a new powder for oral suspension to reduce LDL-C levels in boys and postmenarchal girls aged 10-17 years with heterozygous familial hypercholesterolemia. Colesevelam belongs to the bile acid sequestrant class of drugs. The drug is a non-absorbed, lipid-lowering polymer that was engineered specifically to enhance the binding capacity for bile acids in the intestine, impeding their reabsorption. As the bile acid pool becomes depleted, the hepatic enzyme, cholesterol $7 \alpha$-hydroxylase, is upregulated, thus increasing the conversion of cholesterol to bile acids. This increases demand for cholesterol in the liver cells, resulting in the dual effect of increasing transcription and activity of the cholesterol biosynthetic enzyme, HMG-CoA reductase, and increasing the number of hepatic LDL-C receptors. Two important implications from this study are the reduction of LDL-C by colesevelam, and possible side effects in children. Among adults, colesevelam has been shown to reduce LDL-C by up to $15 \%$. In the current study, it significantly lowered LDL-C by $12.5 \%$. As the drug is approved only for children with LDL-C levels above 190, colesevelam use will not reach target levels. Therefore, the use of colesevelam is of particular value in patients who do not reach their LDL-C target levels with maximal tolerable dosages of statins, and for statin-intolerant patients who may benefit from colesevelam monotherapy. Bile acid sequestrants are not systematically absorbed, and indeed there were no safety or laboratory issues in this study. The drug was well tolerated with relatively good compliance. Gastrointestinal disorders were the most common adverse effects. No significant effects on growth, sexual maturation, fat-soluble vitamin levels, or clotting factors were observed.

\section{Santa Claus: a public health pariah?}

Grills NJa, Halyday $\mathrm{B}^{\mathrm{b}}$

aDepartment of Epidemiology and Preventative Medicine, Monash University, Melbourne, and

bepartment of Health, Collingwood, Vic., Australia

nathangrills@gmail.com

BMJ 2009;339:b5261

It appears that the awareness of Santa Claus, the childhood icon, is near universal. Given Santa's fame, he has considerable potential to influence individual and societal behavior. However, according to the authors, this is not necessarily for the good. Epidemiological studies show there is a correlation between countries that venerate Santa Claus and those that have high levels of childhood obesity. Although the causality has not been proven, it appears that Santa promotes a message that obesity is synonymous with cheerfulness and joviality. The authors therefore offer suggestions to create a supportive environment for Santa's dieting: ceasing the tradition of leaving him cookies, mince pies, milk, brandy or sherry, encouraging him to share the carrots and celery sticks commonly left for Rudolf, and encouraging him to adopt a more active means of delivering toys, such as swapping his reindeer for a bike or simply walking or jogging. From a public health point of view, we now know the causes and have a solution for preventing obesity, the metabolic syndrome, hyperlipidemia and T2DM! 
1. Steinberger J, et al: Progress and challenges in metabolic syndrome in children and adolescents: a scientific statement from the American Heart Association Atherosclerosis, Hypertension, and Obesity in the Young Committee of the Council on Cardiovascular Disease in the Young; Council on Cardiovascular Nursing; and Council on Nutrition, Physical Activity, and Metabolism. Circulation 2009;119:628-647.

2. Simmons RK, et al: The metabolic syndrome: useful concept or clinical tool? Report of a WHO Expert Consultation. Diabetologia 2010;53:600-605.

3. Gidding SS: Familial hypercholesterolemia: a decade of progress. J Pediatr 2010;156:176-177.

4. Meier JJ, Nauck MA: Is the diminished incretin effect in type 2 diabetes just an epi-phenomenon of impaired $\beta$-cell function? Diabetes 2010;59:1117-1125.

5. Pavkov ME, et al: Changing patterns of type 2 diabetes incidence among Pima Indians. Diabetes Care 2007;30:17581763.

6. Sellers EA, et al: Macroalbuminuria and renal pathology in First Nation youth with type 2 diabetes. Diabetes Care 2009;32:786-790.

7. Chong CR, Chabner BA: Mysterious metformin. Oncologist 2009;14:1178-1181.

8. De Kreutzenberg SV, et al: Downregulation of the longevity-associated protein sirtuin-1 in insulin resistance and metabolic syndrome: potential biochemical mechanisms. Diabetes 2010;59:1006-1015.

9. Lewanczuk RZ, Paty BW, Toth EL: Comparison of the $\left[{ }^{13} \mathrm{C}\right]$ glucose breath test to the hyperinsulinemic-euglycemic clamp when determining insulin resistance. Diabetes Care 2004;27:441-447.

10. Friedrichs E: On the pill. Nat Med 2010;16:506-508.

11. Chesney RW: Vitamin D and The Magic Mountain: the anti-infectious role of the vitamin. J Pediatr 2010;156:698-703.

12. Baz-Hecht M, Goldfine AB: The impact of vitamin D deficiency on diabetes and cardiovascular risk. Curr Opin Endocrinol Diabetes Obes 2010;17:113-119.

13. Wang L, et al: Systematic review: vitamin D and calcium supplementation in prevention of cardiovascular events. Ann Intern Med 2010;152:315-323.

14. Pittas AG, et al: Systematic review: vitamin D and cardiometabolic outcomes. Ann Intern Med 2010;152:307-314.

15. Rodriguez W, et al: Improving pediatric dosing through pediatric initiatives: what we have learned? Pediatrics 2008;121:530-539. 